Public Abstract

First Name:Naphtali

Middle Name:Malesela

Last Name:Mokgalapa

Adviser's First Name:Tushar

Adviser's Last Name:Ghosh

Co-Adviser's First Name:Sudarshan

Co-Adviser's Last Name:Loyalka

Graduation Term:SS 2016

Department:Nuclear Engineering

Degree:PhD

\title{
Title:MEASUREMENTS OF ADHESION FORCE BETWEEN SINGLE PARTICLES AND CLUSTERS INTERACTING WITH SOME VHTR STRUCTURAL STEELS
}

The adhesion force and work of adhesion between aerosols (graphite and silver) generated in very high temperature reactors (VHTRs) and interacting with structural materials are presented. The method involves using Atomic Force Microscopy (AFM) in an air environment glove box. The adhesion force data were obtained for a silver particle and a graphite cluster separately interacting with structural materials including Haynes 230, Inconel 617, and Hastelloy X under four different surface conditions including as received and after oxidation for five, 10, and 15 minutes, respectively. It was found that the Johnson, Kendall, and Roberts model (JKR) enabled the prediction of values that were up to three orders of magnitude higher than the experimental data when silver interacted with structural materials. The difference in order of magnitude between experimental data and theory for graphite in particular varied between one and three. In contrast, the inclusion of surface roughness of the silver particle and surface features of structural materials in calculations produced results that were one order of magnitude higher than the experimental data. These comparisons serve to provide insight into the significant influence that surface roughness has on adhesion force. Data produced in this work may be important in understanding the adhesion of silver and reactor grade graphite aerosols to structural materials and can contribute to particle resuspension calculations. 